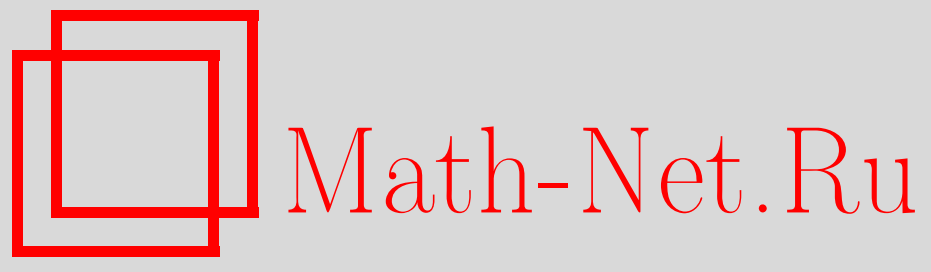

М. А. Горский, Комплексы подслов и 2-усеченные кубы, УМН, 2014, том 69, выпуск 3, 179-180

DOI: https://doi.org/10.4213/rm9596

Использование Общероссийского математического портала Math-Net.Ru подразумевает, что вы прочитали и согласны с пользовательским соглашением http://www . mathnet.ru/rus/agreement

Параметры загрузки:

IP: 54.80 .97 .219

26 апреля 2023 г., 04:22:32

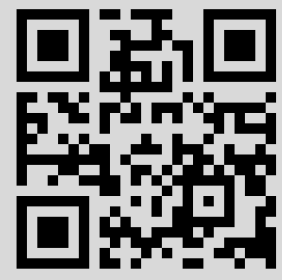




\section{Комплексы подслов и 2-усеченные кубы}

\section{М. А. Горский}

Пусть $W$ - конечная группа Коксетера, $S=\left\{s_{1}, \ldots, s_{n}\right\}-$ множество простых отражений, порождающее $W$. Рассмотрим слово $\mathbf{Q}:=\mathbf{q}_{1} \ldots \mathbf{q}_{m}$ в алфавите простых отражений $\left(\mathbf{q}_{i} \in S \quad \forall i=1, \ldots, m\right)$ и элемент $\pi$ группы $W$. Комплекс подслов $\Delta(\mathbf{Q} ; \pi)$ - это чистый симплициальный комплекс на множестве вершин $\left\{\mathbf{q}_{1}, \ldots, \mathbf{q}_{m}\right\}$, соответствующих буквам (точнее, их позициям) в слове $\mathbf{Q}$. Набор вершин образует симплекс, если дополнение в $\mathbf{Q}$ к соответствующему подслову содержит приведенное выражение $\pi$. Максимальные симплексы отвечают дополнениям приведенных выражений $\pi$ в слове $\mathbf{Q}$. Комплексы подслов были введены А. Кнутсоном и Э. Миллером в работе [1]. Они же показали в [2], что $\Delta(\mathbf{Q} ; \pi)$ является сферическим тогда и только тогда, когда произведение Демазюра слова $\mathbf{Q}$ равно $\pi$; в противном случае $\Delta(\mathbf{Q} ; \pi)$ является триангулированным шаром. Для сферических комплексов подслов важными являются вопросы существования, комбинаторного описания и геометрических реализаций двойственных им многогранников.

В группе $W$ существует и единствен элемент максимальной длины, обозначаемый $w_{o}$. Мы будем рассматривать комплексы подслов вида $\Delta\left(\mathbf{c w}_{o} ; w_{o}\right)$, где с приведенное выражение элемента Коксетера, w ражение $w_{o}$. Эти комплексы допускают реализацию кирпичными многогранниками В. Пило-К. Штумпа [3], которые мы будем обозначать $\mathbf{B}\left(\mathbf{c w}_{o} ; w_{o}\right)$. Для каждого элемента Коксетера $c$ в группе $W$ определены с-кластерный комплекс типа $W$ и двойственный ему многогранник - (обобщенный) c-ассоциаэдр типа $W$. Эти объекты важны в теории кластерных алгебр. С. Чебальос, Ж.-Ф. Лаббе и К. Штумп [4] доказали, что комплексы $\Delta\left(\mathbf{c w}_{o}(\mathbf{c}) ; w_{o}\right)$, где $\mathbf{w}_{o}(\mathbf{c})$ - так называемое $\mathbf{c}$-сортирующее слово для $w_{o}$, являются $c$-кластерными комплексами типа $W$. Следовательно, многогранники $\mathbf{B}\left(\mathbf{c w}_{o}(\mathbf{c}) ; w_{o}\right)$ реализуют $c$-ассоциаэдры типа $W$. Выбор элемента Коксетера $c$ эквивалентен выбору колчана $Q$, являющегося ориентацией диаграммы Коксетера группы $W$. Пусть $\mathbf{c}^{\prime}$ - приведенное выражение произвольного (возможно, равного $c$ ) элемента Коксетера $c^{\prime}$ в группе $W$, и пусть $Q^{\prime}$ - соответствующий колчан. Пусть $\widetilde{Q}_{c, c^{\prime}}$ - колчан, полученный из $Q$ стиранием всех ребер, ориентированных по-разному в $Q$ и в $Q^{\prime}$ (вершины не стираем); $\widetilde{W}_{c, c^{\prime}}-$ группа Коксетера, ориентацией диаграммы которой является $\widetilde{Q}_{c, c^{\prime}}$. Мы можем рассмотреть $c$ как элемент Коксетера в $\widetilde{W}_{c, c^{\prime}}$.

Tеорема 1. $\Delta\left(\mathbf{c w}_{o}\left(\mathbf{c}^{\prime}\right) ; w_{o}\right)$ является с-кластерным комплексом типа $\widetilde{W}_{c, c^{\prime}}$, a $\mathbf{B}\left(\mathbf{c w}_{o}\left(\mathbf{c}^{\prime}\right) ; w_{o}\right)$ реализует соответствующий обобщенный ассоциаэдр. В частносmи, пусть $\mathbf{c}_{\mathrm{rev}}$ обозначает слово $\mathbf{c}$, написанное задом наперед, тогда $\mathbf{B}\left(\mathbf{c w}_{o}\left(\mathbf{c}_{\mathrm{rev}}\right) ; w_{o}\right)$ является (комбинаторным) кубом.

Доказательство получается с помощью аргументов, аналогичных аргументам из работы [4]. Мы используем лемму 2.3 из [4] и проверяем, что все утверждения из [4; раздел 5], кроме леммы 5.5 и предложения 5.6, верны для произвольного приведенного выражения $\mathbf{w}_{o}$, а не только для с-сортирующего слова.

Рассмотрим теперь произвольное приведенное выражение $\mathbf{w}_{o}$ элемента $w_{o}$. Существует биекция $\operatorname{Lr}_{c, \mathbf{w}_{o}}$ между множеством букв слова $\mathbf{c w}_{o}=c_{1} c_{2} \ldots c_{n} w_{1} w_{2} \ldots w_{N}$ и множеством $\Phi_{\geqslant-1}=-\Pi \sqcup \Phi_{+}$почти положительных (т. е. простых отрицательных и всех положительных) корней системы корней $\Phi$, связанной с $W$ :

$$
\operatorname{Lr}\left(c_{i}\right)=-\alpha_{c_{i}} ; \quad \operatorname{Lr}\left(w_{j}\right)=w_{1} w_{2} \ldots w_{j-1}\left(\alpha_{w_{j}}\right),
$$

где $\alpha_{w} \in \Phi-$ корень, соответствующий $w \in W$. Выбор $\mathbf{w}_{o}$ задает полный порядок $<_{\mathbf{w}_{o}}$ на $\Phi_{+}: \alpha<_{\mathbf{w}_{o}} \beta$, если $\operatorname{Lr}^{-1}(\alpha)$ идет в $\mathbf{w}_{o}$ раньше $\operatorname{Lr}^{-1}(\beta)$. Известно, что такой порядок удовлетворяет следующему условию: для любой подсистемы корней ранга 2 в $\Phi$

DOI: $10.4213 / \mathrm{rm} 9596$ 
с каноническими образующими $\alpha, \beta$ либо $a_{1} \alpha+b_{1} \beta<_{\mathbf{w}_{o}} a_{2} \alpha+b_{2} \beta$, если выполнено $a_{1}<a_{2}$, либо $a_{1} \alpha+b_{1} \beta<_{\mathbf{w}_{o}} a_{2} \alpha+b_{2} \beta$, если $a_{1}>a_{2}$. Иначе говоря, положительные корни любой подсистемы ранга 2 упорядочены в одном из двух естественных направлений. Выбор с также задает полный порядок на $\Phi_{+}:<_{\mathbf{c}}=<_{\mathbf{w}_{o}(\mathbf{c})}$. Корень $\gamma \in \Phi_{+}$мы будем называть $\left(c, \mathbf{w}_{o}\right)$-стабильным, если для любой некоммутативной подсистемы $\langle\alpha, \beta\rangle$ ранга 2, содержащей $\gamma$ и такой, что $\gamma \neq \alpha, \beta$, мы имеем

$$
\alpha<\mathbf{c} \beta \Leftrightarrow \alpha<_{\mathbf{w}_{o}} \beta \text {. }
$$

Это условие зависит от $c$, но не от с. Пусть $\operatorname{Stab}\left(\mathbf{c}, \mathbf{w}_{o}\right)-$ множество $\left(c, \mathbf{w}_{o}\right)$-стабильных корней. Основным результатом работы является следующая теорема.

Tеорема 2. (i) Вериины $\Delta\left(\mathbf{c w}_{o} ; w_{o}\right)$ и, эквивалентно, гиперграни $\mathbf{B}\left(\mathbf{c w}_{o} ; w_{o}\right)$ находятся во взаимно однозначном соответствии с простыми отрицательными и (c, $\left.\mathbf{w}_{o}\right)$-стабильными положительными корнями в системе $\Phi$.

(ii) Пусть выражсения $\mathbf{w}_{o}, \mathbf{w}_{o}^{\prime}$ таковы, что $\operatorname{Stab}\left(\mathbf{c}, \mathbf{w}_{o}\right) \subset \operatorname{Stab}\left(\mathbf{c}, \mathbf{w}_{o}^{\prime}\right)$. Тогда комплекс $\Delta\left(\mathbf{c w}_{o}^{\prime} ; w_{o}\right)$ получается из комплекса $\Delta\left(\mathbf{c w}_{o} ; w_{o}\right)$ последовательностью подразбиений ребер. Аналогично, многогранник $\mathbf{B}\left(\mathbf{c w}_{o}^{\prime} ; w_{o}\right)$ получается из многогранника $\mathbf{B}\left(\mathbf{c w}_{o} ; w_{o}\right)$ последовательностъю усечений граней коразмерности 2.

Доказательство опирается на результаты работы [5], где было показано, как движения кос в группе $W$ индуцируют композиции подразбиений ребер и обратных операций на комплексах подслов. Далее, мы показываем, что найдутся два корня $\alpha$, $\beta \in \operatorname{Stab}\left(\mathbf{c}, \mathbf{w}_{o}\right), \alpha<_{\mathbf{w}_{o}} \beta, \beta<_{\mathbf{w}_{o}^{\prime}} \alpha$, между которыми (относительно порядка $<_{\mathbf{w}_{o}}$ ) нет других корней из $\operatorname{Stab}\left(\mathbf{c}, \mathbf{w}_{o}\right)$. Остается показать, что движениями кос можно переставить буквы, соответствующие корням из отрезка $[\alpha, \beta]_{\mathbf{w}_{o}}$, так, чтобы $\beta$ шло раньше $\alpha$. В качестве перестановки можно взять с-сортирующее слово, тогда результат получается из свойства слов для группы $W$.

Класс 2-усеченных кубов, т. е. многогранников, получающихся из куба последовательностью усечений граней коразмерности 2, обладает интересными свойствами и включает в себя важные семейства многогранников (см. [6]). Каждый 2-усеченный куб является флаговым многогранником (т. е. любое множество его гиперграней имеет непустое пересечение, как только любая пара из этого множества имеет непустое пересечение). Из теорем 1 и 2 мы получаем следующее утверждение.

СлЕДСТвИЕ 1. Любой многогранник вида $\mathbf{B}\left(\mathbf{c w}_{o} ; w_{o}\right)$ является 2-усеченным кубом и, следовательно, флаговым. В частности, любой обобщенный ассочиаэдр является 2-усеченным кубом.

\section{Список литературы}

[1] A. Knutson, E. Miller, Ann. of Math. (2), 161:3 (2005), 1245-1318. [2] A. Knutson, E. Miller, Adv. Math., 184:1 (2004), 161-176. [3] V. Pilaud, C. Stump, Brick polytopes of spherical subword complexes: a new approach to generalized associahedra, 2011 (v3-2014), 52 pp., arXiv: 1111.3349. [4] C. Ceballos, J.-P. Labbé, C. Stump, J. Algebraic Combin., 39:1 (2014), 17-51. [5] M. Gorsky, Subword complexes and edge subdivisions, 2013, 12 c., arXiv: 1305.5499; М. Горский, “Комплексы подслов и подразбиения ребер”, Тр. МИАН (в печати). [6] V. M. Buchstaber, V. D. Volodin, Associahedra, Tamari lattices, and related structures, Tamari memorial Festschrift, Progr. Math., 299, Birkhäuser, Basel, 2012, 161-186.

Михаил Александрович Горский

(Mikhail A. Gorsky)

Математический институт им. В. А. Стеклова РАН

E-mail: mike.gorsky@gmail.com
Представлено В. М. Бухштабером Принято редколлегией 28.05.2014 